\title{
REINGRESO AL EMPRENDIMIENTO DESPUÉS DEL FRACASO: FACTORES QUE INFLUYEN SOBRE LOS EMPRENDEDORES SERIALES Y DE SEGUNDA OPORTUNIDAD
}

\author{
RE-ENTRY INTO ENTREPRENEURSHIP AFTER FAILURE: FACTORS \\ INFLUENCING SERIAL AND SECOND CHANCE ENTREPRENEURS \\ María José Ibáñez Caamaño ${ }^{\mathrm{a}}$ \\ Trabajo empírico - investigación \\ Recibido: 21-abril-2020 / Revisado: 10-junio-2020 / Aceptado: 11-junio-2020
}

\section{Resumen}

Este artículo investiga los factores que influyen en la probabilidad de volver a emprender luego del fracaso empresarial, entre estos, los factores sociodemográficos y los económicos. En particular, se examina el efecto de la edad, el nivel educacional, el rol proveedor del emprendedor en la familia, la cobertura de los instrumentos y los servicios públicos para el emprendimiento, así como los motivos que llevan al emprendedor al cierre del negocio, sobre la disposición del emprendedor fallido a iniciar una nueva empresa. Se utiliza una muestra de 274 emprendedores fallidos de Chile, el país con la mayor tasa de actividad emprendedora (TEA) en el mundo, según el reporte del GEM 2019/2020. Los resultados de este estudio señalan que las variables principales tienen una influencia negativa en la probabilidad de reingresar al emprendimiento luego del fracaso empresarial, pero solo resultan significativas la edad, la cobertura regional de los servicios técnicos para el emprendimiento, la quiebra, la preferencia por el trabajo asalariado y el desempleo a nivel regional. Esta investigación contribuye a ampliar la evidencia empírica respecto a los factores que influyen sobre los emprendedores en serie y de segunda oportunidad, más allá de los aspectos relativos a la intención emprendedora y los elementos del comportamiento del emprendedor. Este trabajo entrega conclusiones relevantes orientadas al diseño de políticas públicas e instrumentos de fomento para los emprendedores que están dispuestos a crear un nuevo negocio luego del fracaso empresarial.

Palabras clave: emprendimiento serial, fracaso empresarial, reingreso, ecosistema emprendedor.

\begin{abstract}
This article investigates factors that influence the probability of re-starting after business failure, including sociodemographic and economic variables. Particularly, it examines the effect of age, educational level, the entrepreneur's role as provider in the family, the coverage of public instruments and services for entrepreneurship and the reasons that lead the entrepreneur to close the business, on the willingness of the failed entrepreneur to start a new business. It uses a sample of 274 failed entrepreneurs from Chile, the country with the highest rate of Total early-stage Entrepreneurial Activity (TEA) in the world, according to the GEM 2019/2020 report. The results of this study indicate that the main variables have a negative influence on the probability of re-entering entrepreneurship after business failure, but only age, regional coverage of technical services for entrepreneurship, bankruptcy, preference for wage-employment and the level of regional unemployment are significant. This research contributes to broadening the empirical evidence regarding the factors that influence serial and second-chance entrepreneurs, beyond aspects related to entre-
\end{abstract}

a Universidad del Desarrollo. Concepción, Chile. Correo electrónico: maribanezc@udd.cl 
preneurial intent and elements of entrepreneurial behavior. This paper provides relevant conclusions for the design of public policies and support instruments for entrepreneurs who are willing to create a new business after business failure.

Keywords: Serial entrepreneurship, business failure, reentry, entrepreneurship ecosystem.

\section{Introducción}

Se ha argumentado ampliamente en la literatura que la actividad emprendedora está relacionada de forma positiva con el desarrollo económico de los países y su crecimiento económico (Acs, Estrin, Mickiewicz y Szerb, 2018; Kasseeah, 2016; Peprah y Adekoya, 2020). Esto resalta la importancia del estudio de la actividad emprendedora en todas sus dimensiones como un campo de investigación multidisciplinario (Bruyat y Julien, 2001; Chandra, 2018; Jones, Coviello y Tang, 2011; López-Fernández, Serrano-Bedia y Pérez-Pérez, 2016).

Entre los aspectos más investigados en emprendimiento se encuentra el estudio de la intención de emprender (Karimi et al., 2017; Zhao, Seibert y Hills, 2005), las características de los emprendedores (Karimi et al., 2017; Kot, Meyer y Broniszewska, 2016; Lecuna, Cohen y Chávez, 2017) y el fracaso de las nuevas empresas (Artinger y Powell, 2016; Khelil, 2016). Un área naciente en esta disciplina se refiere a qué ocurre con los emprendedores luego del fracaso empresarial (Corner, Singh y Pavlovich, 2017) y la decisión de iniciar una nueva empresa luego de fallar (Lafontaine y Shaw, 2016). Estos aspectos del emprendimiento abren nuevas oportunidades para expandir este campo de investigación.

Se ha comprobado que ciertos emprendedores se ven afectados de forma negativa luego del fracaso empresarial en términos sociales (Simmons, Wiklund y Levie, 2014), psicológicos (Byrne y Shepherd, 2015) y financieros (Shepherd, Wiklund y Haynie, 2009). Sin embargo, existe escasa evidencia respecto a por qué los emprendedores podrían tener intención de emprender luego de fracasar (Lafontaine y Shaw, 2016).

Este estudio examina variables demográficas y estructurales que pueden influir en la intención de los emprendedores de iniciar un nuevo negocio luego de fracasar en su anterior actividad empresarial, basado en una muestra de 274 emprendedores que fracasaron en su actividad empresarial y que declaran su intención de volver a emprender o no. Entender el fenómeno del emprendimiento en serie y el emprendimiento de segunda oportunidad es esencial en el propósito de formular políticas públicas que permitan dar soporte a este tipo de emprendedores (Tipu, 2020).
Esta investigación contribuye de forma importante a la literatura. Primero, permite profundizar en la magnitud de emprendedores que están dispuestos a iniciar un nuevo negocio luego de fracasar y, especialmente, conocer más acerca de sus características y los motivos del fracaso de sus negocios. Segundo, identifica las variables demográficas que influyen en la probabilidad de que un emprendedor vuelva a formar una empresa luego de fallar. Tercero, determina si ciertos aspectos del ecosistema emprendedor a nivel regional tienen influencia en la probabilidad de reemprender.

Este artículo explora, en primer lugar, los antecedentes teóricos de la intención emprendedora, el fracaso empresarial y el emprendimiento serial. Luego se describe la metodología y los instrumentos utilizados. En la sección de resultados, se describen los principales hallazgos del estudio y se analizan respecto a las hipótesis planteadas. Luego, se discuten los resultados de acuerdo con la literatura previa y se concluye respecto a los hallazgos más relevantes. Por último, se comentan las limitaciones e implicaciones del estudio y se plantean posibles temas para futuras investigaciones.

\section{Antecedentes teóricos}

La intención de emprender (Karimi et al., 2016; Zhao et al., 2005), las características de los emprendedores y del ecosistema emprendedor (Karimi et al., 2017; Kot et al., 2016; Lecuna et al., 2017; Spigel, 2017) y el fracaso de las nuevas empresas (Artinger y Powell, 2016; Khelil, 2016) han colmado la literatura en el área de emprendimiento, llevándola a convertirse en uno de los campos de investigación más populares del último tiempo (Chandra, 2018; Meyer et al., 2014; Shane, 2012). El enfoque positivo respecto al emprendimiento como facilitador del desarrollo y el crecimiento económico (Acs et al., 2018; Kasseeah, 2016; Peprah y Adekoya, 2020) ha llevado a muchos países con economías emergentes a desplegar recursos de todo tipo para fortalecer los ecosistemas para el emprendimiento (Simmons, Wiklund, Levie, Bradley y Sunny, 2019; Spigel, 2017) y estimular la actividad emprendedora.

El discurso exitista de la actividad emprendedora se ha complementado con el estudio del fracaso empresarial de los emprendimientos nacientes, llevando a obtener 
poderosas conclusiones respecto a los factores de éxito y fracaso de las empresas de nueva creación (Chatterjee y Das, 2016; Dimov y De Clercq, 2006; Pardo y Alfonso, 2017; Song, Podoynitsyna, Van Der Bij y Halman, 2008). Sin embargo, la pregunta acerca de lo que ocurre con los emprendedores luego del fracaso empresarial aún persiste. En este sentido, se han hecho valiosas contribuciones respecto a las consecuencias psicológicas, sociales y financieras negativas del fracaso para los emprendedores (Byrne y Shepherd, 2015; Shepherd y Cardon, 2009; Simmons et al., 2014) y su capacidad de reponerse a los aspectos adversos de fallar (Hayward, Forster, Sarasvathy y Fredrickson, 2010; Korber y McNaughton, 2018; Simmons, Carr, Hsu y Shu, 2016). En este contexto se enmarca el estudio respecto a los emprendedores en serie (Baù, Sieger, Eddleston y Chirico, 2017).

Aunque el campo del emprendimiento ha sido ampliamente explorado en los últimos años, aún no existe una definición unificada de emprendimiento o de emprendedor (Bridge, 2017). Según el Global Entrepreneurship Monitor (GEM), emprendimiento es "cualquier intento de crear una nueva empresa comercial o expandir una empresa existente por un individuo, un equipo de individuos o un negocio establecido" (Zacharakis, Bygrave y Shepherd, 2000). Esta definición está en línea con la importancia que la literatura atribuye al estudio de la intención de emprender, sin embargo, la investigación de la intención emprendedora depende del contexto y de la perspectiva que adopte el investigador (Schlaegel y Koenig, 2014). Por tanto, esta línea de investigación ha tomado diversos caminos, con diferentes aproximaciones teóricas y metodológicas, y en algunos casos conclusiones opuestas (Liñán y Fayolle, 2015; Mård, 2020; Shinnar, Giacomin y Janssen, 2012). Los estudios sobre la decisión de establecer un negocio abarcan variables demográficas, económicas y de percepción (atención a las oportunidades, el miedo al fracaso y la confianza en las propias habilidades) (Arenius y Minniti, 2005). Sin embargo, las conclusiones se restringen a los emprendedores nacientes, ya que la información acerca de los emprendedores que han fracasado es difícil de conseguir para los investigadores.

La investigación sobre el emprendimiento se centra, predominantemente, en el éxito (Singh, Corner y Pavlovich, 2007), sin embargo, es común que las empresas fracasen (Shepherd, Patzelt y Wolfe, 2011). En Chile, cerca del $7 \%$ de los emprendimientos fracasan en su etapa inicial (Guerrero y Serey, 2018); a nivel general nacional, en el 2016, se registraron 3593 empresas con quiebras terminadas (SIR, 2016). El fracaso en los negocios es parte de la dinámica empresarial y depende de múltiples factores (Alaka et al., 2016). Así como se estudian los factores que determinan el éxito empresarial (Chatterjee y Das, 2016), los determinantes del fracaso en los negocios han sido estudiados en el área del emprendimiento, por ejemplo, los ingresos insuficientes para la supervivencia, los problemas de financiamiento, los problemas con la ejecución del plan estratégico, las crisis económicas o políticas y la falta de indicadores o seguimiento (Pardo y Alfonso, 2017). Existe asociación positiva entre el fracaso empresarial y el desarrollo y la evolución de los ecosistemas de emprendimiento: los emprendedores que han experimentado un fracaso pueden tener un valor particular para impulsar la evolución de los ecosistemas emprendedores (Simmons et al., 2019). La naturaleza de la experiencia empresarial puede determinar la forma en que los empresarios se adaptan (Ucbasaran, Westhead, Wright y Flores, 2010), e influir sobre la posibilidad de que un emprendedor comience un nuevo negocio luego de haber fallado. Esta visión positiva, desde el punto de vista sistémico, encuentra su contracara en las consecuencias negativas que el fracaso empresarial tiene para algunos emprendedores a nivel individual (Jenkins, Wiklund y Brundin, 2014; Shepherd et al., 2011). No todos los emprendedores que han fallado son capaces de superar el fracaso y continuar con sus vidas con normalidad (Corner et al., 2017).

Estudios argumentan que los emprendedores que fracasan experimentan una diversidad de emociones negativas (Shepherd et al., 2011; 2009), como, por ejemplo, frustración, angustia, decepción, preocupación y vergüenza, entre otras. Así, entonces, es particularmente llamativo que ciertas personas superen estas emociones negativas y vuelvan a intentar establecer un negocio a pesar del fracaso (Corner et al., 2017). Esta capacidad de recuperación, también llamada resiliencia (Korber y McNaughton, 2018), por lo general, se atribuye al exceso de confianza de algunos emprendedores y a sus actitudes hacia el fracaso (Hayward et al., 2010). Las habilidades cognitivas que caracterizan a estos empresarios para procesar sus salidas comerciales también desempeñan un papel importante en sus intenciones de emprender una actividad empresarial en serie (Simmons et al., 2016).

Los emprendedores que abren negocios repetidamente, llamados también emprendedores seriales, son más comunes de lo que se cree. Un estudio realizado en Texas reveló que, para el 2011, el 25.6\% de las empresas del Estado eran operadas por emprendedores en serie (Lafontaine y Shaw, 2016). Otro trabajo realizado en Europa afirma que, para el 2010 , entre el $18 \%$ y el $30 \%$ de los emprendedores eran emprendedores seriales (Plehn-Dujowich, 2010). Lo que hace que los emprendedores fracasados vuelvan a intentarlo es una pregunta de investigación interesante e importante que no ha recibido 
mucha atención en la literatura (Baù et al., 2017). Existe evidencia de que ocurre un proceso de acumulación de experiencia y aprendizaje cada vez que el emprendedor fracasa y vuelve a intentarlo (Guerrero y Peña-Legazkue, 2019; Hsu, Shinnar, Powell y Coffey, 2017). Incluso, se ha comprobado que las empresas de emprendedores en serie pueden ser más exitosas que las de emprendedores nacientes (Shaw y Sørensen, 2019) y se ha argumentado que dar espacio y oportunidad a los empresarios fracasados para que vuelvan a surgir permitiría potenciar las actividades empresariales y mejorar el desarrollo económico en los países con economías emergentes (Amankwah-Amoah, 2018).

\section{Desarrollo de hipótesis}

Desde la perspectiva del emprendedor naciente existe un vínculo entre ciertas variables demográficas y económicas y la decisión de empezar un negocio (Arenius y Minniti, 2005). En el caso de los emprendedores en serie se han descrito relaciones diversas entre la edad, el sexo y la carrera profesional del emprendedor fallido y su decisión de volver a intentarlo (Baù et al., 2017; Simmons et al., 2019).

Las personas más jóvenes tienen más probabilidades de comenzar una nueva empresa que las personas mayores, en términos de emprendimientos nacientes (Levesque y Minniti, 2006). En este estudio se comprueba si se mantiene esta condición sobre la probabilidad de que el emprendedor fallido reingrese al emprendimiento.

H1: a mayor edad del emprendedor fallido, menor es la probabilidad de volver a crear un negocio.

Una dimensión que no ha sido explorada es el contexto familiar del emprendedor en serie y cómo puede influir el rol de proveedor o proveedora de la familia en la decisión de volver a crear un negocio después de haber fallado. Se ha comprobado que existe relación positiva entre el reconocimiento y el apoyo familiar hacia el emprendedor y su decisión de emprender (Rambe, 2019). Sin embargo, para el emprendedor o emprendedora que cumple el rol de proveedor en la familia puede ser difícil movilizar los recursos financieros necesarios para afrontar las pérdidas del negocio fallido y, además, realizar inversiones en una nueva empresa, debido a la dependencia económica de otros miembros de la familia.

H2: los emprendedores fallidos que son proveedores o proveedoras del hogar tienen menor probabilidad de volver a crear un negocio.
Los empresarios en serie cuya empresa anterior fracasó, probablemente culpen al entorno externo (Eggers y Song, 2015). Los factores institucionales y regulatorios tienen influencia sobre la intención de emprender (Morales-Alonso, Pablo-Lerchundi y Núñez Del Río, 2016), y tienden a ser diferentes a niveles regionales, así como las percepciones culturales del fracaso empresarial (Cardon, Stevens y Potter, 2011). Una fuerte infraestructura para el emprendimiento y la disminución de la burocracia influyen de forma positiva en la actividad emprendedora naciente se comprueba si esto también es válido para la intención de reemprender.

H3: altos niveles de inversión pública en desarrollo de nuevos negocios aumentan la probabilidad de que los emprendedores fallidos estén dispuestos a crear un nuevo negocio.

Los motivos para abandonar un emprendimiento pueden ser variados: jubilación, quiebra, conflictos legales, problemas de salud, cierre planificado o preferencia por un trabajo asalariado, entre otros. Todos estos casos se consideran fracasos empresariales, dado que no se produce la permanencia de la empresa en el tiempo (PlehnDujowich, 2010). Diferente es el caso de las fusiones, adquisiciones y cambios de giro, pues estas circunstancias no están contempladas como fracasos empresariales (INE, 2020). La quiebra es la razón de abandono de la empresa más traumática (SIR, 2016). En el caso de las empresas pequeñas, los problemas de quiebra son especialmente difíciles, puesto que no hay ningún negocio que valga la pena salvar y no hay activos por los que luchar, por tanto, es la situación del emprendedor la que realmente preocupa (Baird y Morrison, 2005). Debido a las consecuencias psicológicas, sociales y financieras negativas del fracaso para los emprendedores (Byrne y Shepherd, 2015; Shepherd y Cardon, 2009; Simmons et al., 2014), es menos probable que los emprendedores fallidos lo intenten de nuevo.

H4: el cierre del negocio por motivos de quiebra reduce la probabilidad de que el emprendedor fallido esté dispuesto a empezar un nuevo negocio.

\section{Metodología}

Para este estudio se utilizan datos de la Sexta Encuesta de Microemprendimiento (EME6), publicada en el 2020 por el Instituto Nacional de Estadísticas (INE) de Chile (INE, 2020). La muestra incluye 274 emprendedores fallidos chilenos, es decir, emprendedores cuyas empresas deja- 
ron de funcionar en el 2018. Se consideran emprendedores fallidos puesto que las razones de la desaparición de sus empresas no están relacionadas con una fusión, adquisición $u$ otra figura colaborativa.

Los emprendedores de la muestra fueron separados por zonas geográficas a fin de incorporar las medidas de inversión pública en ayuda financiera y soporte dirigido a emprendimiento en cada una de las 15 regiones de Chile de las que existen datos. Como proxy de la ayuda financiera se utilizó la cantidad de proyectos de emprendimiento aprobados para financiamiento público a través de instrumentos de la Corporación de Fomento (Corfo), y como aproximación al soporte e infraestructura pública para el emprendimiento se utilizó la cantidad de oficinas del Servicio de Cooperación Tecnológica (Sercotec); ambas variables se construyeron a nivel regional con información pública de ambos servicios (Corfo, 2020a; Sercotec, 2020) y se ponderaron por la cantidad de habitantes en cada una de las regiones, correspondientes al 2018. La mayor parte de los emprendedores que tienen intención de reemprender se encuentra en la zona central del país, que contempla las regiones V, VI, VII y VIII e incluye a la Región Metropolitana. Coincidentemente, la mayor cantidad de oficinas Sercotec se encuentran en la zona central, donde se concentra casi el 57\% del total de la cobertura nacional. Del total de proyectos financiados por Corfo, el $66 \%$ de estos emprendimientos se ubican en la zona central y casi el 40\% solo en la Región Metropolitana.

Los datos se analizan, primero, en un nivel descriptivo, y luego se utiliza un modelo probit para la estimación de la probabilidad de elección de volver a crear un negocio luego del fracaso empresarial (Train, 2009). Mediante el test de exogeneidad de Wald se determinó que no existen problemas de endogeneidad en el modelo propuesto y, por tanto, no se requiere el uso de variables instrumentales (Stock y Watson, 2012). Las definiciones conceptuales y codificación/operacionalización de las variables, así como su rol en el modelo se describen en la tabla 1.

Tabla 1. Definiciones conceptuales y codificación/operacionalización de las variables

\begin{tabular}{|c|c|c|}
\hline Variables & Definición conceptual & Codificación/operacionalización \\
\hline \multicolumn{3}{|l|}{ Variable dependiente } \\
\hline Reingreso & $\begin{array}{l}\text { Intención del emprendedor fallido de iniciar una nueva } \\
\text { empresa. }\end{array}$ & $\begin{array}{l}0=\text { No } \\
1=\text { Sí }\end{array}$ \\
\hline \multicolumn{3}{|l|}{ Variables independientes } \\
\hline Edad & Edad del emprendedor fallido en tramos. & $\begin{array}{l}1=\text { Entre } 15 \text { y } 24 \text { años } \\
2=\text { Entre } 25 \text { y } 34 \text { años } \\
3=\text { Entre } 35 \text { y } 44 \text { años } \\
4=\text { Entre } 45 \text { y } 54 \text { años } \\
5=\text { Entre } 55 \text { y } 64 \text { años } \\
6=65 \text { años o más }\end{array}$ \\
\hline Nivel educacional & Mayor nivel educacional alcanzado por el emprendedor. & $\begin{array}{l}1=\text { Sin educación formal } \\
2=\text { Educación básica } \\
3=\text { Educación media } \\
4=\text { Educación superior técnica } \\
5=\text { Educación superior universitaria } \\
6=\text { Postítulo o magíster }\end{array}$ \\
\hline Proveedor & $\begin{array}{l}\text { Condición/rol de soporte financiero principal de la } \\
\text { familia del emprendedor o emprendedora. }\end{array}$ & $\begin{array}{l}0=\text { No es proveedor } \\
1=\text { Es proveedor }\end{array}$ \\
\hline Cobertura apoyo financiero & $\begin{array}{l}\text { Cantidad de proyectos de emprendimiento financiados } \\
\text { con recursos públicos a través de Corfo a nivel regional } \\
\text { en el } 2018 \text {. }\end{array}$ & $\begin{array}{l}\text { Ratio de cobertura, calculado como la división entre el número de } \\
\text { proyectos financiados y la cantidad de personas que habitan la región. }\end{array}$ \\
\hline Cobertura soporte técnico & $\begin{array}{l}\text { Cantidad de Centros de Negocios de apoyo al emprendi- } \\
\text { miento de Sercotec a nivel regional en } 2018 .\end{array}$ & $\begin{array}{l}\text { Ratio de cobertura, calculado como la división entre el número de } \\
\text { Centro de Negocios y la cantidad de personas que habitan la región. }\end{array}$ \\
\hline Quiebra & $\begin{array}{l}\text { El cierre de la empresa se debió a que el negocio no } \\
\text { generó ingresos suficientes. }\end{array}$ & $\begin{array}{l}0=\text { No } \\
1=\text { Sí }\end{array}$ \\
\hline \multicolumn{3}{|l|}{ Variables de control } \\
\hline Oportunidad laboral & $\begin{array}{l}\text { El cierre de la empresa se debió a que el emprendedor } \\
\text { consiguió un trabajo asalariado. }\end{array}$ & $\begin{array}{l}0=\text { No } \\
1=\text { Sí }\end{array}$ \\
\hline Género & Género con el cual se identifica el emprendedor. & $\begin{array}{l}0=\text { Mujer } \\
1=\text { Hombre }\end{array}$ \\
\hline Desempleo & $\begin{array}{l}\text { Cantidad de desocupados en edad de trabajar en la región } \\
\text { en } 2018 .\end{array}$ & Tasa de desocupación regional \\
\hline
\end{tabular}


Se utilizan de forma adicional cuatro variables de control: género, tasa de desempleo regional, nivel educacional y si el motivo del cierre del negocio fue que el emprendedor consiguió un trabajo asalariado. Se han descubierto brechas de género en el emprendimiento serial (Simmons et al., 2019) y existe una asociación positiva entre el desempleo y el emprendimiento en serie, en cuanto a las expectativas salariales del individuo para la elección ocupacional (Audretsch, Carree, Thurik y Van Stel, 2005; Plehn-Dujowich, 2010). La educación tiene una influencia primaria, especialmente cuando el conocimiento adquirido y las habilidades desarrolladas se relacionan directamente con la implementación práctica de una actividad independiente (Păunescu, Popescu y Duennweber, 2018). Se encuentra también evidencia respecto a que existen ciertas condiciones para la sustitución entre la condición de emprendedor y trabajador asalariado en términos de la actitud hacia el riesgo de los emprendedores (Vereshchagina y Hopenhayn, 2009), por tanto, es pertinente incluir estas variables en el presente estudio.

\section{Resultados}

En la tabla 2 se presentan los estadísticos descriptivos para la muestra tanto para las variables categóricas como para las variables continuas. Los emprendedores que están dispuestos a volver a empezar un negocio luego de fracasar representan cerca del $70 \%$ de la muestra. Un aspecto llamativo de los datos son las bajas tasas de cobertura regional de la ayuda financiera entregada por Corfo y de la infraestructura de apoyo al emprendimiento de Sercotec, las cuales se encuentran muy por debajo del $1 \%$ de cobertura regional, contrario a la noción de una fuerte institucionalización del emprendimiento en Chile (Chandra y Medrano-Silva, 2012). La mayoría de los emprendedores fallidos tienen entre 45 y 64 de edad, y cerca del $45 \%$ de los emprendedores que han fracasado solo completaron la enseñanza media. E1 32\% de los emprendedores fallidos declaran la quiebra como motivo de fracaso.

Tabla 2. Estadísticos descriptivos

\begin{tabular}{l|c|c}
\hline \multicolumn{1}{c|}{ Variables categóricas } & Frecuencia & Porcentaje \\
\hline Reingreso & & \\
\hline $0=$ No & 83 & 30.29 \\
\hline $1=$ Sí & 191 & 69.71 \\
\hline Edad & & \\
\hline $1=$ Entre 15 y 24 años & 26 & 9.49 \\
\hline $2=$ Entre 25 y 34 años & 45 & 16.42 \\
\hline
\end{tabular}

(Continúa)

\begin{tabular}{|c|c|c|}
\hline Variables categóricas & Frecuencia & Porcentaje \\
\hline $3=$ Entre 35 y 44 años & 43 & 15.69 \\
\hline $4=$ Entre 45 y 54 años & 72 & 26.28 \\
\hline $5=$ Entre 55 y 64 años & 65 & 23.72 \\
\hline $6=65$ años o más & 23 & 8.39 \\
\hline \multicolumn{3}{|l|}{ Nivel educacional } \\
\hline $1=$ Sin educación formal & 6 & 2.19 \\
\hline 2 = Educación básica & 74 & 27.01 \\
\hline $3=$ Educación media & 123 & 44.89 \\
\hline 4 = Educación superior técnica & 25 & 9.12 \\
\hline 5 = Educación superior universitaria & 44 & 16.06 \\
\hline $6=$ Postítulo o magíster & 2 & 0.73 \\
\hline \multicolumn{3}{|l|}{ Proveedor } \\
\hline $0=$ No es proveedor & 125 & 45.62 \\
\hline $1=$ Es proveedor & 149 & 54.38 \\
\hline \multicolumn{3}{|l|}{ Quiebra } \\
\hline $0=\mathrm{No}$ & 187 & 68.25 \\
\hline $1=$ Sí & 87 & 31.75 \\
\hline \multicolumn{3}{|l|}{ Oportunidad laboral } \\
\hline $0=\mathrm{No}$ & 163 & 59.49 \\
\hline $1=$ Sí & 111 & 40.51 \\
\hline \multicolumn{3}{|l|}{ Género } \\
\hline $0=$ Mujer & 95 & 34.67 \\
\hline $1=$ Hombre & 179 & 65.33 \\
\hline Variables continuas & Media & Desv. Est. \\
\hline Cobertura apoyo financiero & 0.0000284 & 0.0000142 \\
\hline Cobertura soporte técnico & $3.54 \mathrm{e}-06$ & $2.51 \mathrm{e}-06$ \\
\hline Desempleo & 6.673358 & 1.414026 \\
\hline Observaciones & 274 & \\
\hline
\end{tabular}

En la tabla 3 se reportan los resultados del análisis probit y en la tabla 4 los efectos marginales y el nivel de correcta clasificación del modelo. Se comprueba que existe una relación negativa entre la condición de proveedor o proveedora familiar y la probabilidad de iniciar un nuevo negocio luego del fracaso. Sin embargo, esta relación no es significativa, por tanto, no es posible aceptar la hipótesis 1. La relación entre la edad del emprendedor fallido y su intención de reemprender es negativa y significativa, por tanto, se acepta la hipótesis 2 . Con respecto a la hipótesis 3 , se encontró que a mayor infraestructura regional para el emprendimiento menor es la probabilidad de 
Tabla 3. Coeficientes estimados análisis Probit

\begin{tabular}{|c|c|c|}
\hline Variable & Coeficiente & Desv. Est. \\
\hline Edad & $-0.1480417^{* *}$ & 0.0698716 \\
\hline Proveedor & -0.0413116 & 0.1898363 \\
\hline Cobertura apoyo financiero & 723.0327 & 7281.098 \\
\hline Cobertura soporte técnico & $-90255.83^{* *}$ & 44326.94 \\
\hline Quiebra & $-0.5608677 * *$ & 0.2288884 \\
\hline Oportunidad laboral & $-0.6481149 * * *$ & 0.2246175 \\
\hline Género & 0.2406863 & 0.185198 \\
\hline Desempleo & $-0.1291512 *$ & 0.0751231 \\
\hline Nivel educacional & -0.0794611 & 0.0840081 \\
\hline Constante & $2.812003 * * *$ & 0.7408348 \\
\hline Pseudo R-Cuadrado & 0.0688 & \\
\hline Observaciones & 274 & \\
\hline
\end{tabular}

Notas: *Significativo al $10 \%$; **Significativo al $5 \%$; ***Significativo al $1 \%$.

iniciar un nuevo negocio después de haber fallado, por tanto, se rechaza esta hipótesis. Un argumento a favor de este resultado algo contradictorio puede ser que los Centros de Negocio Sercotec están orientados a emprendedores nacientes más que emprendedores seriales y, en general, los programas estatales para el emprendimiento están enfocados en emprendedores novatos (Chandra y Medrano-Silva, 2012).
Se comprueba la hipótesis 4 , el cierre del negocio a causa de que la empresa no generó ingresos suficientes es negativa y significativa, es decir, aquellos emprendedores fallidos que han experimentado la quiebra tendrán menor probabilidad de emprender de nuevo con relación a los emprendedores que han cerrado sus negocios por otras causas, lo cual puede ser una muestra de miedo al fracaso (Morgan y Sisak, 2016).

Con respecto a las variables de control, aquella referida al cierre del negocio porque el emprendedor encontró un trabajo asalariado resultó ser significativa y negativa. Los emprendedores que han dejado su negocio por una oportunidad laboral tienen menos probabilidad de convertirse en emprendedores en serie, incluso esta variable tiene mayor impacto que la referida al motivo de cierre por quiebra. Esto entrega información respecto a las preferencias de estabilidad laboral de los emprendedores y su actitud hacia el riesgo (Vereshchagina y Hopenhayn, 2009). La variable de desempleo resultó ser significativa, a menor nivel, con una influencia negativa en la probabilidad de emprender después del fracaso empresarial, es decir, mayores niveles de desempleo regional reducen la probabilidad de convertirse en emprendedores en serie, al contrario de los hallazgos en otros estudios referidos al emprendimiento naciente. Audretsch et al. (2005) proponen que existe una relación compleja entre el desempleo y el emprendimiento, en el sentido de que su asociación depende del ciclo de negocios. No obstante, en sus resultados empíricos se encuentra una relación positiva entre el desempleo y el emprendimiento, en el caso de la actividad empresarial naciente. En este estudio, los resultados

Tabla 4. Efectos marginales dy/dx, análisis Probit

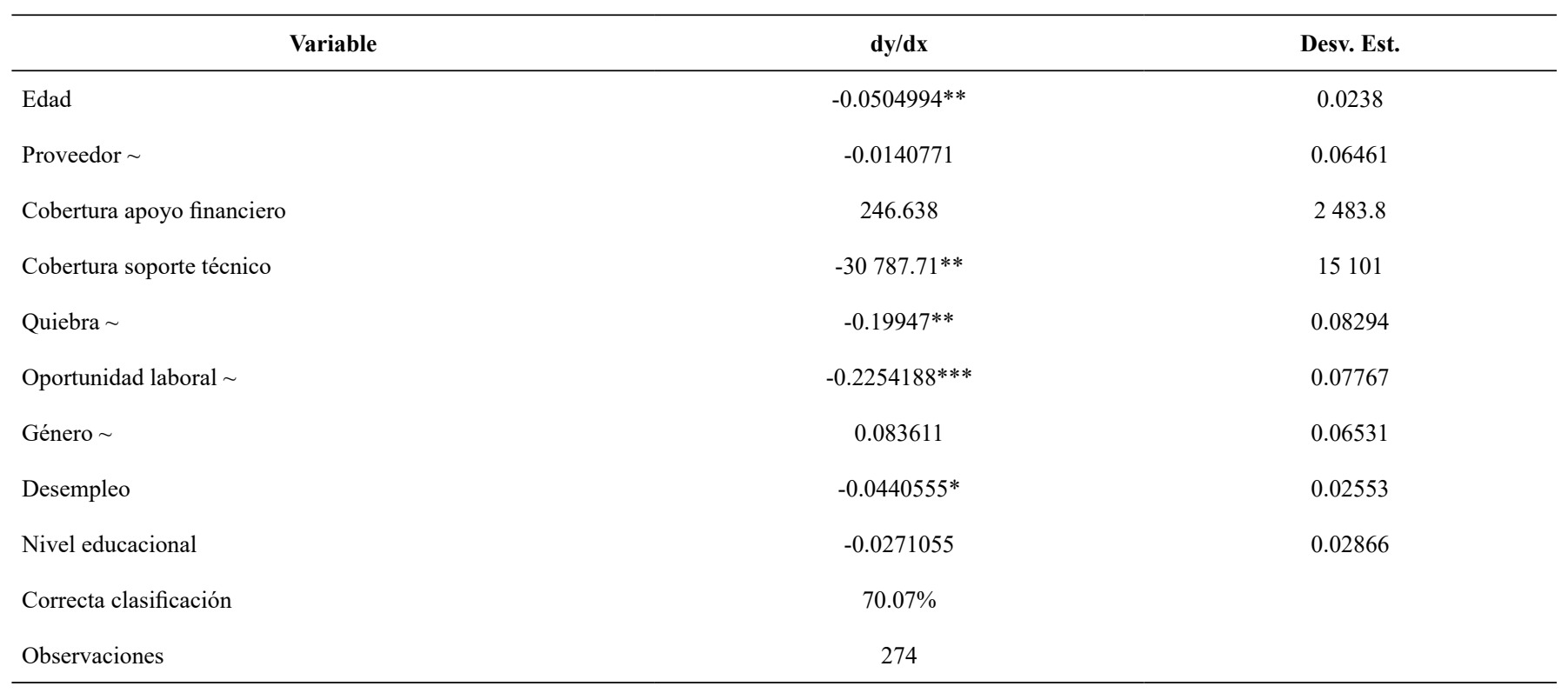

Notas: $(\sim)$ dy/dx es para cambios discretos en la variable binaria de 0 a $1 .{ }^{*}$ Significativo al $10 \% ; * *$ Significativo al $5 \% ; * * *$ Significativo al $1 \%$. 
indican que la dinámica entre el desempleo y el reemprendimiento es distinta.

\section{Discusión y conclusiones}

El concepto de emprendedor en serie por el cual se define a los emprendedores que abren negocios repetidamente es cuestionable desde varias perspectivas. La primera tiene que ver con la relación que existe entre el nivel de riqueza del emprendedor y su decisión de emprender (Vereshchagina y Hopenhayn, 2009), cuál es el nivel de riqueza inicial que le permite a un emprendedor abrir negocios repetidamente, cuánta riqueza debe acumular de sus empresas antiguas para dar lugar a las nuevas, o debe abandonar antiguos proyectos a fin de tener la capacidad financiera de abrir otros nuevos. Posiblemente, estas reflexiones tienen más que ver con un enfoque de cartera que con la disposición a emprender luego de un fracaso empresarial (Plehn-Dujowich, 2010). La segunda cuestión se relaciona con el aprendizaje que se obtiene del fracaso empresarial (Guerrero y Peña-Legazkue, 2019; Hsu et al., 2017), lo cual, se ha argumentado, puede mejorar el desempeño de emprendimientos posteriores (Shaw y Sørensen, 2019). Sin embargo, el fracaso empresarial puede corregir el sesgo cognitivo de riesgo mal percibido del emprendedor y frenar las intenciones de emprender nuevamente luego de fallar (Overall, 2016); aquí se debe hacer una importante distinción entre los emprendedores en serie y los emprendedores de segunda oportunidad.

Los primeros no necesariamente estarán relacionados con el fracaso empresarial, mientras que los segundos sí. En el caso de los emprendedores en serie, estos podrían acumular aprendizaje infinitamente mientras cuenten con los recursos para operar más y más proyectos. Los emprendedores de segunda oportunidad, en cambio, podrían utilizar el aprendizaje del fracaso empresarial del primer intento para no fallar en el segundo y considerar el segundo emprendimiento su última oportunidad de tener éxito, y en caso de fracasar no intentarlo nuevamente. La última reflexión es relativa a la distinción entre la intención de emprender y la intención de reemprender (Tipu, 2020). La intención de emprender está referida, principalmente, a emprendedores novatos (Karimi et al., 2017), pero también podría aplicarse a los emprendedores en serie que no han experimentado fracasos empresariales. Por su parte, la intención de emprender luego de un fracaso empresarial abarca dimensiones diferentes (Tipu, 2020). Por tanto, puede haber menor coincidencia entre la intención de reemprender después del fracaso y la cantidad de emprendimientos de segunda oportunidad, con relación a la intención de emprender y la cantidad de emprendimientos nacientes.
En este estudio se detectó un alto nivel de intención de reemprender entre los emprendedores de la muestra, pues casi el $70 \%$ de ellos estaba dispuesto a iniciar un nuevo negocio luego de haber fracasado en el anterior. Al indagar en estos resultados se determinó que solo el $28 \%$ de estos emprendedores había experimentado la quiebra, y este motivo de fracaso tuvo una influencia negativa y significativa en la probabilidad de volver a crear una empresa luego del fracaso. Esto apoya las conclusiones de Corner et al. (2017) respecto a que no es un patrón común que las personas superen estas experiencias negativas y resta importancia a la influencia positiva de la acumulación de aprendizaje en el fracaso empresarial (Hsu et al., 2017). Por otra parte, de los emprendedores dispuestos a iniciar un nuevo negocio luego de fracasar con el anterior, más del 38\% había abandonado su empresa por un puesto de trabajo asalariado. Se estableció una relación negativa y significativa entre la oportunidad laboral y la probabilidad de crear una empresa luego de fracasar, lo que señala cómo existe un efecto de sustitución entre el emprendimiento y el trabajo asalariado, es decir, las personas privilegian la estabilidad laboral por sobre la expectativa de triunfar en los negocios luego de fracasar (Vereshchagina y Hopenhayn, 2009). Los resultados respecto a los motivos del fracaso empresarial y la intención de crear una nueva empresa revelan que, efectivamente, existe una corrección en la percepción del riesgo y una modificación de la conducta del emprendedor luego del fracaso empresarial (Overall, 2016), lo que ya se había planteado en términos de la distinción entre intención emprendedora e intención de reemprender.

Se ha argumentado cómo los emprendedores que han experimentado un fracaso pueden tener un valor particular para impulsar la evolución de los ecosistemas emprendedores (Simmons et al., 2019). En este estudio se examinó la influencia del apoyo estatal por medio de financiamiento e infraestructura para el emprendimiento, en la probabilidad de que los emprendedores vuelvan a crear un negocio luego de fallar. Esta aproximación a la estructura del ecosistema para el emprendimiento evidencia que la cobertura de la ayuda económica a los emprendedores no tiene influencia significativa, más aún, un mayor nivel de cobertura en el apoyo técnico a los emprendedores tiene un efecto negativo y significativo en la probabilidad de reemprender. Se comprobó que la mayoría de los instrumentos de financiamiento y apoyo al emprendimiento están dirigidos a emprendedores nacientes (Corfo, 2020b), por tanto, el efecto de estas coberturas para el emprendedor en serie o de segunda oportunidad es mínimo. Si se añade, además, que muchas instituciones financieras poseen estrictos requisitos para el otorgamiento de créditos que incluyen la evaluación de la 
experiencia empresarial anterior del emprendedor, entonces el ecosistema para el emprendimiento serial o de segunda oportunidad no se encuentra lo suficientemente desarrollado por ahora, al menos en lo que respecta a los elementos estructurales o institucionales (Spigel, 2017). Esto reafirma la importancia del nivel de riqueza del emprendedor que le permite ser emprendedor serial o de segunda oportunidad, ya que ante la dificultad de conseguir apoyo financiero externo deberá afrontar la pérdida del negocio anterior y la inversión en el nuevo mediante fuentes alternativas o recursos propios.

Baù et al. (2017) argumentan que la edad tiene una relación no lineal con la probabilidad de reingresar al emprendimiento, bajo un enfoque de desarrollo profesional. Este argumento es poderoso en el caso en que los emprendedores posean expectativas de carrera profesional. Sin embargo, no todos los emprendedores tienen los niveles de educación necesarios para el desarrollo de una carrera profesional y, en algunos casos, las etapas en el desarrollo de la carrera no tienen relación con la edad, sino más bien con el acceso a oportunidades. En este estudio la edad tiene una influencia negativa y significativa en la probabilidad de reentrada en el emprendimiento, en línea con lo plateado por Levesque y Minniti (2006). Se comprobó a través de una extensión del modelo general, usando como variable de compensación el nivel educacional de los emprendedores, y no se encontraron resultados significativos para describir la relación entre la edad, el nivel educacional y la probabilidad de emprender luego de un fracaso en el sentido que propone Baù et al. (2017).

\section{Limitaciones e implicaciones}

Una limitación para el estudio del reingreso al emprendimiento luego del fracaso empresarial radica en la escasez de información respecto a los emprendedores que han fallado. En su mayoría, estos emprendedores desaparecen de las observaciones en las encuestas de emprendimiento, las cuales, en general, no son longitudinales. Para este tipo de estudio es importante realizar una distinción entre los emprendedores por necesidad y de oportunidad. puesto que sí existe una fuerte sustitución entre las oportunidades de trabajo asalariado y la intención de emprender después del fracaso empresarial podríamos estar frente a emprendimientos de paso, es decir, empresas que son creadas para percibir ingresos mientras se espera la inserción en el mercado laboral formal como asalariado.

La presente investigación utiliza datos de Chile, el país que presenta la tasa más alta de actividad emprendedora en estado temprano (TEA) en el mundo, en el 2019, según el Global Entrepreneurship Monitor (GEM)
(Bosma et al., 2020). Por tanto, podrían existir notorias diferencias en las conclusiones de un estudio similar en otros países del mundo con menores tasas de actividad emprendedora.

A fin de entender mejor el rol de los emprendedores fallidos en el ecosistema emprendedor es necesario contar con mejores variables relativas a los aspectos estructurales, regulatorios e institucionales que caracterizan a estos ecosistemas del contexto empresarial general. Cualquier estudio que no incluya todas las dimensiones del ecosistema emprendedor (Spigel, 2017) estará siempre incompleto. Sin embargo, existe dificultad para operacionalizar ciertas variables y, en este sentido, sería interesante combinar métodos cualitativos y cuantitativos para una aproximación más profunda al problema.

Los resultados sugieren que no existe una dimensión integrada al ecosistema emprendedor que incluya a los emprendedores en serie o de segunda oportunidad. En este sentido, es importante diseñar políticas públicas e instrumentos de fomento especialmente dirigidos a emprendedores que han fracasado y están dispuestos a intentarlo de nuevo. Así, el debate puede fluir en tres direcciones: la primera relacionada con el desarrollo de instrumentos orientados a evitar el fracaso empresarial o reducir las pérdidas mediante un cierre planificado del negocio, como, por ejemplo, programas de apoyo para emprendimientos de bajo crecimiento o desempeño deficiente; una segunda directiva puede ser incluir al reemprendimiento en la cartera de proyectos elegibles para el financiamiento estatal a través de Corfo o garantía estatal dirigida a créditos de la banca privada; por último, implementar una estrategia comunicacional que promueva la resiliencia como una característica deseable en los emprendedores, suavizando el discurso exitista acerca de la actividad emprendedora. Por ahora, se han adaptado las normativas relativas a la quiebra con el fin de facilitar el cierre de negocios fallidos y mitigar las pérdidas de sus propietarios (SIR, 2016). Sin embargo, es necesario poner atención no solo a las consecuencias financieras negativas del emprendimiento fallido, sino también a los costos psicológicos y sociales que afectan al emprendedor fracasado.

\section{Referencias}

Acs, Z. J., Estrin, S., Mickiewicz, T., \& Szerb, L. (2018). Entrepreneurship, institutional economics, and economic growth: An ecosystem perspective. Small Business Economics, 51(2), 501-514.

Alaka, H. A., Oyedele, L. O., Owolabi, H. A., Ajayi, S. O., Bilal, M., \& Akinade, O. O. (2016). Methodological approach of construction business failure predic- 
tion studies: A review. Construction Management and Economics, 34(11), 808-842.

Amankwah-Amoah, J. (2018). Revitalising serial entrepreneurship in Sub-Saharan Africa: Insights from a newly emerging economy. Technology Analysis \& Strategic Management, 30(5), 499-511.

Arenius, P., \& Minniti, M. (2005). Perceptual variables and nascent entrepreneurship. Small Business Economics, 24(3), 233-247.

Artinger, S., \& Powell, T. C. (2016). Entrepreneurial failure: Statistical and psychological explanations. Strategic Management Journal, 37(6), 1047-1064.

Audretsch, D. B., Carree, M. A., Thurik, R., \& Van Stel, A. J. (2005). Does self-employment reduce unemployment? Journal of Business Venturing, 23(6), 673-686.

Baird, D. G., \& Morrison, E. R. (2005). Serial entrepreneurs and small business bankruptcies. Columbia Law Review, 105, 2310-2368.

Baù, M., Sieger, P., Eddleston, K. A., \& Chirico, F. (2017). Fail but try again? The effects of age, gender, and multiple-owner experience on failed entrepreneurs' reentry. Entrepreneurship Theory and Practice, 41(6), 909-941.

Bosma, N., Hill, S., Ionescu-Somers, A., Kelley, D., Levie, J., \& Tarnawa, A. (2020). GEM 2019/2020 Global Report. Gem Mundial 2019/2020. Recuperado de https:// www.gemconsortium.org/file/open?fileId $=50443$

Bridge, S. (2017). Does 'entrepreneurship'exist? Small Enterprise Research, 24(2), 206-213.

Bruyat, C., \& Julien, P.-A. (2001). Defining the field of research in entrepreneurship. Journal of Business Venturing, 16(2), 165-180.

Byrne, O., \& Shepherd, D. A. (2015). Different strokes for different folks: Entrepreneurial narratives of emotion, cognition, and making sense of business failure. Entrepreneurship Theory and Practice, 39(2), 375-405.

Cardon, M. S., Stevens, C. E., \& Potter, D. R. (2011). Misfortunes or mistakes?: Cultural sensemaking of entrepreneurial failure. Journal of Business Venturing, 26(1), 79-92.

Chandra, A., \& Medrano-Silva, M. A. (2012). Business incubation in Chile: Development, financing and financial services. Journal of Technology Management \& Innovation, 7(2), 1-13.

Chandra, Y. (2018). Mapping the evolution of entrepreneurship as a field of research (1990-2013): A scientometric analysis. PloS One, 13(1), 1-24.

Chatterjee, N., \& Das, N. (2016). A study on the impact of key entrepreneurial skills on business success of Indian micro-entrepreneurs: A case of Jharkhand region. Global Business Review, 17(1), 226-237.
Corfo. (2020a). Página principal. Recuperado de https:// www.corfo.cl/sites/cpp/home

Corfo. (2020b). Programas y convocatorias. Recuperado de https://www.corfo.cl/sites/cpp/programas-y-convo catorias

Corner, P. D., Singh, S., \& Pavlovich, K. (2017). Entrepreneurial resilience and venture failure. International Small Business Journal, 35(6), 687-708.

Dimov, D., \& De Clercq, D. (2006). Venture capital investment strategy and portfolio failure rate: A longitudinal study. Entrepreneurship Theory and Practice, 30(2), 207-223.

Eggers, J. P., \& Song, L. (2015). Dealing with failure: Serial entrepreneurs and the costs of changing industries between ventures. Academy of Management Journal, 58(6), 1785-1803.

Guerrero, M., \& Peña-Legazkue, I. (2019). Renascence after post-mortem: The choice of accelerated repeat entrepreneurship. Small Business Economics, 52(1), 47-65.

Guerrero, M., \& Serey, T. (2018). Reporte Nacional de Chile 2018. Global Entrepreneurship Monitor.

Hayward, M. L. A., Forster, W. R., Sarasvathy, S. D., \& Fredrickson, B. L. (2010). Beyond hubris: How highly confident entrepreneurs rebound to venture again. Journal of Business Venturing, 25(6), 569-578.

Hsu, D. K., Shinnar, R. S., Powell, B. C., \& Coffey, B. S. (2017). Intentions to reenter venture creation: The effect of entrepreneurial experience and organizational climate. International Small Business Journal, 35(8), 928-948.

INE. (2020). Sexta encuesta de microemprendimiento. Recuperado de https://www.economia.gob.cl/2020/03 /11/sexta-encuesta-de-microemprendimiento-eme6. htm

Jenkins, A. S., Wiklund, J., \& Brundin, E. (2014). Individual responses to firm failure: Appraisals, grief, and the influence of prior failure experience. Journal of Business Venturing, 29(1), 17-33.

Jones, M. V., Coviello, N., \& Tang, Y. K. (2011). International entrepreneurship research (1989-2009): A domain ontology and thematic analysis. Journal of Business Venturing, 26(6), 632-659.

Karimi, S., Biemans, H. J. A., Lans, T., Chizari, M., \& Mulder, M. (2016). The impact of entrepreneurship education: A study of Iranian students' entrepreneurial intentions and opportunity identification. Journal of Small Business Management, 54(1), 187-209.

Karimi, S., Biemans, H. J. A., Naderi Mahdei, K., Lans, T., Chizari, M., \& Mulder, M. (2017). Testing the relationship between personality characteristics, contex- 
tual factors and entrepreneurial intentions in a developing country. International Journal of Psychology, 52(3), 227-240.

Kasseeah, H. (2016). Investigating the impact of entrepreneurship on economic development: A regional analysis. Journal of Small Business and Enterprise Development, 23(3), 896-916.

Khelil, N. (2016). The many faces of entrepreneurial failure: Insights from an empirical taxonomy. Journal of Business Venturing, 31(1), 72-94.

Korber, S., \& McNaughton, R. B. (2018). Resilience and entrepreneurship: A systematic literature review. International Journal of Entrepreneurial Behavior \& Research, 24(7), 1129-1154.

Kot, S., Meyer, N., \& Broniszewska, A. (2016). A crosscountry comparison of the characteristics of Polish and South African woman entrepreneurs. Economics and Sociology, 9(4), 207-221.

Lafontaine, F., \& Shaw, K. (2016). Serial entrepreneurship: Learning by doing? Journal of Labor Economics, 34(S2), S217-S254.

Lecuna, A., Cohen, B., \& Chávez, R. (2017). Characteristics of high-growth entrepreneurs in Latin America. International Entrepreneurship and Management Journal, 13(1), 141-159.

Levesque, M., \& Minniti, M. (2006). The effect of aging on entrepreneurial behavior. Journal of Business Venturing, 21(2), 177-194.

Liñán, F., \& Fayolle, A. (2015). A systematic literature review on entrepreneurial intentions: Citation, thematic analyses, and research agenda. International Entrepreneurship and Management Journal, 11(4), 907-933.

López-Fernández, M. C., Serrano-Bedia, A. M., \& Pérez-Pérez, M. (2016). Entrepreneurship and family firm research: A bibliometric analysis of an emerging field. Journal of Small Business Management, 54(2), 622-639.

Mård, M. (2020). Book review: A research agenda for entrepreneurial cognition and intention. Londres: SAGE Publications.

Meyer, M., Libaers, D., Thijs, B., Grant, K., Glänzel, W., \& Debackere, K. (2014). Origin and emergence of entrepreneurship as a research field. Scientometrics, 98(1), 473-485.

Morales-Alonso, G., Pablo-Lerchundi, I., \& Núñez Del Río, M.-C. (2016). Intención emprendedora de los estudiantes de ingeniería e influencia de factores contextuales. Revista de Psicología Social, 31(1), 75-108.

Morgan, J., \& Sisak, D. (2016). Aspiring to succeed: A model of entrepreneurship and fear of failure. Journal of Business Venturing, 31(1), 1-21.
Overall, J. (2016). The dark side of entrepreneurship: A conceptual framework of cognitive biases, neutralization, and risky entrepreneurial behaviour. Academy of Entrepreneurship Journal, 22(2), 1-12.

Pardo, C., \& Alfonso, W. (2017). Applying “attribution theory" to determine the factors that lead to the failure of entrepreneurial ventures in Colombia. Journal of Small Business and Enterprise Development, 24(3), 562-584.

Păunescu, C., Popescu, M. C., \& Duennweber, M. (2018). Factors determining desirability of entrepreneurship in Romania. Sustainability, 10(11), 3893, 1-22.

Peprah, A. A., \& Adekoya, A. F. (2020). Entrepreneurship and economic growth in developing countries: Evidence from Africa. Business Strategy and Development.

Plehn-Dujowich, J. (2010). A theory of serial entrepreneurship. Small Business Economics, 35(4), 377-398.

Rambe, P. (2019). The influence of selected personal demographic and institutional variables on venture creation: A case of small technology-oriented ventures in an emerging economy. African Journal of Science, Technology, Innovation and Development, 11(1), 13-28.

Schlaegel, C., \& Koenig, M. (2014). Determinants of entrepreneurial intent: A meta-analytic test and integration of competing models. Entrepreneurship Theory and Practice, 38(2), 291-332.

Sercotec. (2020). Página principal. Recuperado de https:// www.sercotec.cl/

Shane, S. (2012). Delivering on the promise of entrepreneurship as a field of research. Academy of Management Review, 37(1), 10-20.

Shaw, K., \& Sørensen, A. (2019). The productivity advantage of serial entrepreneurs. ILR Review, 72(5), 1225-1261.

Shepherd, D. A., \& Cardon, M. S. (2009). Negative emotional reactions to project failure and the self-compassion to learn from the experience. Journal of Management Studies, 46(6), 923-949.

Shepherd, D. A., Patzelt, H., \& Wolfe, M. (2011). Moving forward from project failure: negative emotions, affective commitment, and learning from the experience. Academy of Management Journal, 54(6), 1229-1259.

Shepherd, D. A., Wiklund, J., \& Haynie, J. M. (2009). Moving forward: Balancing the financial and emotional costs of business failure. Journal of Business Venturing, 24(2), 134-148.

Shinnar, R. S., Giacomin, O., \& Janssen, F. (2012). Entrepreneurial perceptions and intentions: The role of gender and culture. Entrepreneurship Theory and Practice, 36(3), 465-493. 
Simmons, S. A., Carr, J. C., Hsu, D. K., \& Shu, C. (2016). The regulatory fit of serial entrepreneurship intentions. Applied Psychology, 65(3), 605-627.

Simmons, S. A., Wiklund, J., \& Levie, J. (2014). Stigma and business failure: Implications for entrepreneurs' career choices. Small Business Economics, 42(3), 485-505.

Simmons, S. A., Wiklund, J., Levie, J., Bradley, S. W., \& Sunny, S. A. (2019). Gender gaps and reentry into entrepreneurial ecosystems after business failure. Small Business Economics, 53(2), 517-531.

Singh, S., Corner, P., \& Pavlovich, K. (2007). Coping with entrepreneurial failure. Journal of Management \& Organization, 13(4), 331-344.

SIR. (2016). Boletín Estadístico de Quiebras. Chile: Superintendencia de Insolvencia y Reemprendimiento.

Song, M., Podoynitsyna, K., Van Der Bij, H., \& Halman, J. I. M. (2008). Success factors in new ventures: A meta-analysis. Journal of Product Innovation Management, 25(1), 7-27.

Spigel, B. (2017). The relational organization of entrepreneurial ecosystems. Entrepreneurship: Theory and Practice, 41(1), 49-72.
Stock, J., \& Watson, M. (2012). Introducción a la econometría ( $3^{\mathrm{a}}$ ed.). Madrid: Pearson Educación.

Tipu, S. A. A. (2020). Entrepreneurial reentry after failure: A review and future research agenda. Journal of Strategy and Management, 13(2), 198-220.

Train, K. E. (2009). Discrete choice methods with simulation ( $2^{\mathrm{a}}$ ed.). Nueva York: Cambridge University Press.

Ucbasaran, D., Westhead, P., Wright, M., \& Flores, M. (2010). The nature of entrepreneurial experience, business failure and comparative optimism. Journal of Business Venturing, 25(6), 541-555.

Vereshchagina, G., \& Hopenhayn, H. A. (2009). Risk taking by entrepreneurs. American Economic Association, 99(5), 1808-1830.

Zacharakis, A., Bygrave, W. D., \& Shepherd, D.A. (2000). Global entrepreneurship monitor: National entrepreneurship assessment, United States of America: 2000 executive Report. Kauffman Center for Entrepreneurial Leadership at the Ewing Marion Kauffman.

Zhao, H., Seibert, S. E., \& Hills, G. E. (2005). The mediating role of self-efficacy in the development of entrepreneurial intentions. Journal of Applied Psychology, 90(6), 1265. 